Z. Klin. Chem. Klin. Biochem.

13. Jg. 1975, S. 31-35

\title{
Eine enzymatische Methode zur Isolierung von Tubuli und Zellen aus menschlicher Nierenrinde
}

\author{
Von H. Bojar, K. Balzer, F. Boeminghaus und W. Staib
}

Aus dem Institut für Physiologische Chemie II (Direktor Prof. Dr.W. Staib) und der Urologischen Klinik (Direktor Prof. Dr. H. Dettmar) der Universität Düsseldorf

(Eingegangen am 14. Juni/10. September 1974)

Herrn Prof. Dr. Karl Hinsberg zum 80. Geburtstag gewidmet

Menschliche Nierentubuli und Zellen wurden nach einem enzymatischen Verfahren aus Nierenrindenschnitten mit Kollagenase isoliert. Die hohe Ausbeute von etwa 80 \% des Feuchtgewichts der zur Isolierung inkubierten Schnitte gestattete es, eine Vielzahl von Experimenten unter identischen Ausgangsbedingungen durchzuführen.

Als Funktionskriterium für die Vitalität der isolierten Zellen wurde die Gluconeogenese aus verschiedenen Substraten bestimmt. Die höchsten Syntheseraten wurden in Gegenwart von Fructose erzielt. Aus Lactat wurde mehr Glucose als aus Pyruvat gebildet. Mit Prolin und Glutamin ließen sich nur geringe Gluconeogeneseraten erreichen. In Gegenwart von $10 \mathrm{mmol} / 1$ Pyruvat stieg die Glucosebildung bis zur 80. Minute linear an. Ado- $3^{\prime}: 5^{\prime}-\mathrm{P}$ stimulierte in $10 \mu$ molarer Konzentration die Glucosebildung aus Lactat, während sie durch 1 mmolare, 0,1 mmolare und 1 mmolare Konzentrationen gehemmt wurde.

\section{An enzymatic method for the isolation of tubules and cells from human kidney cortex}

The isolation of tubules and cells from human kidney cortex was realized by an enzymatic method. Tubules and cells were released from slices of kidney cortex by collagenase. The yield amounted to $80 \%$ of the wet weight of incubated cortex slices. Thus numerous experiments with isolated tubules from one organ could be performed.

Glucose production from different substrates was measured in order to test the biochemical integrity of the isolated cells. The highest rates of glucose formation were obtained with fructose as precursor. Glucose production was higher from lactate than from pyruvate. With proline and glutamine as substrates only small amounts of glucose were produced. Glucose formation from $10 \mathrm{mmol} / 1 \mathrm{pyruvate}$ was linear with time up to 80 minutes. Ado-3':5'-P stimulated glucose formation at $10 \mu$ molar concentration and inhibited gluconeogenesis at $1 \mathrm{mmolar}, 0.1 \mathrm{mmolar}$ and $1 \mu \mathrm{molar}$ concentrations.

Für die Untersuchung des normalen und pathologisch veränderten Stoffwechsels menschlicher Nierenrinden wird ein in vitro Modell benötigt, das intaktes Gewebe in großer Ausbeute für eine Vielzahl von Experimenten liefert. Darüberhinaus ist eine reproduzierbare Dosierbarkeit mit einem entsprechend kleinen Variationskoeffizienten zu fordern. Die Nierenperfusion hat den Nachteil, daß man an einem Organ nur eine sehr begrenzte Anzahl von Parametern untersuchen kann. Weiterhin wird die Interpretation der Perfusionsergebnisse z. B. bei Untersuchungen der Gluconeogenese durch die Heterogenität des Organs (gluconeogenetisch aktive Rinde, glykolysierendes Mark) erschwert. Stoffwechseluntersuchungen an Nierenschnitten werden kompliziert durch eine limitierte Sauerstoff- und Substratdiffusion in das' Gewebe (1), durch zeitraubendes Abwiegen der Schnitte für verschiedene Inkubationen und durch einen hohen Variationskoeffizienten.
Für verschiedene tierische Organe sind enzymatische Techniken zur Zellisolierung entwickelt worden (2, 3, 4). Kollagenase erwies sich als Enzym der Wahl, um aus Rattenlebern morphologisch und biochemisch intakte Pareichymzellen mit erhaltenen Hormonrezeptoren auf der äußeren Zellmembran in hoher Ausbeute zu gewinnen $(5,6)$.

Die Isolierung von Tubuli und Zellen mittels Kollagenase aus Rinden exstirpierter Nieren erwachsener Personen ist Gegenstand der vorliegenden Untersuchung.

\section{Material und Methoden}

\section{Material}

Die Versuche wurden an fünf menschlichen Nieren durchgeführt, die wegen verschiedener Indikationen entfernt werden mußten. In drei Fällen lagen hypernephroide Nierenkarzinome vor, die auf den oberen Pol des Organs beschränkt waren. 
Pararenale sowie pulmonale Metastasen fanden sich nicht. Die Gewebsproben wurden aus histologisch gesichert tumorfreier Nierenrinde entnommen. Bei zwei weiteren Organen handelte es sich um eine Harnstauungsniere bzw. eine pyelonephritische Niere. Das Alter der Patienten lag zwischen 45 und 74 Jahren. Vier Patienten waren normalgewichtig, einer adipös. Bei allen lag ein befriedigender Allgemeinzustand vor. Die Werte für Harnstoff, Kreatinin, Kalium, Natrium, Calcium und die des Blutbildes lagen im Normbereich. Nur bei einer Patientin fanden sich Zeichen eines Harnwegsinfektes. Die durchschnittliche Anoxiezeit der Nieren während der Operation betrug 15 Minuten. Bis zum Beginn der Präparation wurden die Organe weitere 15 Minuten in eiskaltem Krebs-Ringer-Phosphat-Puffer aufbewahrt.

\section{Isolierungsmethode}

Nach Dekapsulation wurde das Nierenmark entfernt und von der verbliebenen Rinde mit dem Handmikrotom nach Stadie \& Riggs (7) Schnitte ( $350 \mu \mathrm{m})$ angefertigt, die in calciumfreiem Krebs-Ringer-Phosphat-Puffer aufgenommen und gewogen wurden. Danach überführten wir sie mit dem fünffachen Volumen (w/v) calciumfreien Krebs-Ringer-Bicarbonat-Puffer in $100 \mathrm{ml}$ Polyäthylenflaschen. Nach Zugabe von Kollagenase (Endkonzentration $10 \mathrm{mg} / \mathrm{g}$ Schnitteinwaage) wurden die Flaschen mit Carbogen $\left(95 \% \mathrm{O}_{2}, 5 \% \mathrm{CO}_{2}\right)$ begast, verschraubt und in einem Schüttelbad (Frequenz 180/min, Amplitude $2,5 \mathrm{~cm}) 20$ Minuten bei $37^{\circ} \mathrm{C}$ inkubiert. Nach Zugabe von Calciumchlorid (Endkonzentration $5 \mathrm{mmol} / \mathrm{l}$ ) wurde die Inkubation 50 Minuten fortgesetzt. Anschließend wurde die Suspension in einen $50 \mathrm{ml}$ Standzylinder überführt. Das enzymatisch nicht aufgeschlossene Gewebe sedimentierte innerhalb 20 Sekunden. Der Überstand wurde $1 \mathrm{~min}$ bei $50 \mathrm{~g}$ in einer Kühlzentrifuge (Sorvall RC2-B, Rotor SS-34) zentrifugiert und anschließend zweimal mit Krebs-Ringer-Phosphat-Puffer gewaschen. Das Sediment wurde zur Bestimmung des Feuchtgewichtes gewogen und in dem 9-fachen Volumen (w/v) KrebsRinger-Bicarbonat-Puffer aufgenommen.

\section{Versuchsdurchführung}

Jeweils $0,4 \mathrm{ml}$ dieser Suspension wurden in $10 \mathrm{ml}$ Polyäthylenröhrchen (Packard Instrument GmbH, Frankfurt) zu 1,6 ml Krebs-Ringer-Bicarbonat-Puffer pipettiert, der die angegebenen Substrate bzw. Ado-3': $5^{\prime}-\mathrm{P}$ enthielt. Die Röhrchen wurden mit Carbogen begast, verschraubt und in einem Schüttelbad
(Frequenz $120 / \mathrm{min}$, Amplitude $2,5 \mathrm{~cm}$ ) bei $37^{\circ} \mathrm{C}$ inkubiert. Mit $0,1 \mathrm{ml}$ einer $300 \mathrm{~g} / 1$ Perchlorsäure wurde die Reaktion gestoppt. Nach 2 minütiger Zentrifugation (Eppendorf Tischzentrifuge 3200) wurde der Utberstand dekantiert und mit Kaliumhydrogencarbonat in Substanz neutralisiert.

Rindenschnitte (350 $\mu \mathrm{m}, 40-60 \mathrm{mg}$ Feuchtgewicht) wurden in $2 \mathrm{ml}$ Krebs-Ringer-Bicarbonat-Puffer mit Lactat $(10 \mathrm{mmol} / \mathrm{l})$ wie oben inkubiert und weiterverarbeitet.

\section{Chemikalien und Reagenzien}

Wenn nicht anders angegeben, wurden Chemikalien der Firma Merck, Darmstadt, vom Reinheitsgrad p.a. verwendet. Die Glucose bestimmten wir mit Testkombinationen der Firma Boehringer Mannheim, von der wir auch Ado-3': $5^{\prime}$-P bezogen. Kollagenase (CLS II) der Firma Worthington Biochemical Corporation, Freehold, New Yersey, USA wurde benutzt.

\section{Statistik}

Die statistische Auswertung erfolgte nach dem t-Test nach Student.

\section{Ergebnisse}

Für die Isolierung wurden durchschnittlich $5 \mathrm{~g}$ Rindenschnitte eingesetzt. Daraus ließen sich nach unserem Verfahren im Mittel $4 \mathrm{~g}$ Tubuli und Zellen isolieren; das entspricht einer Ausbeute von $80 \%$.

Abbildung 1 zeigt eine phasenkontrastmikroskopische Aufnahme isolierter menschlicher Nierentubuli und Zellen. Ein Teil der Tubuli wurde durch das Isolierungsverfahren weiter dissoziiert, so daß sich in der Suspension auch alle $Z$ wischenstufen vom isolierten Tubulus bis zu Einzelzellen fanden (Abb. 2). Daneben ließen sich vereinzelt Glomerula nachweisen.

Zur Beurteilung der Reproduzierbarkeit der Methodik wurden die Variationskoeffizienten der Gluconeogenese

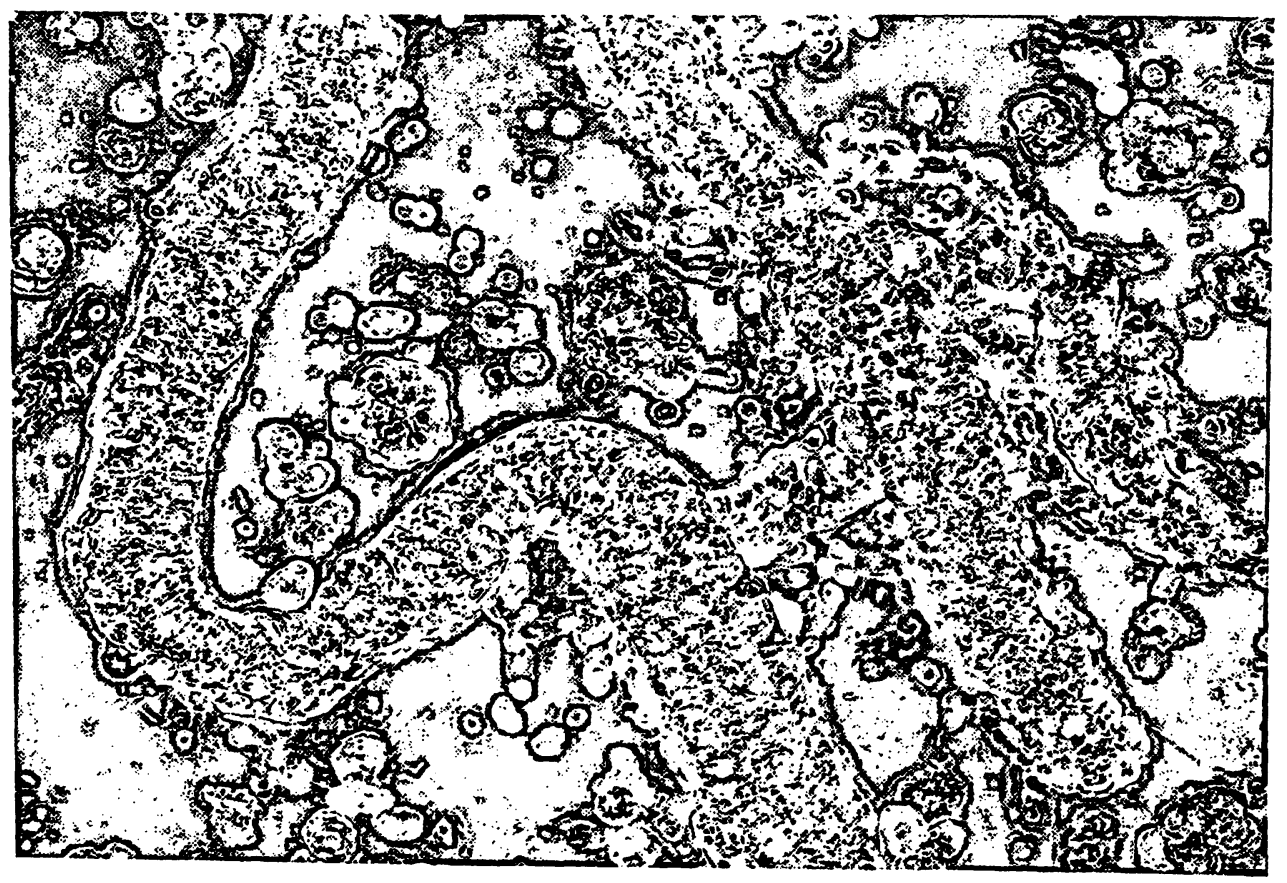

Abb. 1. Phasenkontrastmikroskopische Aufnahme isolierter menschlicher Nierentubuli und Zellen. Vergrößerung etwa 400 fach. 


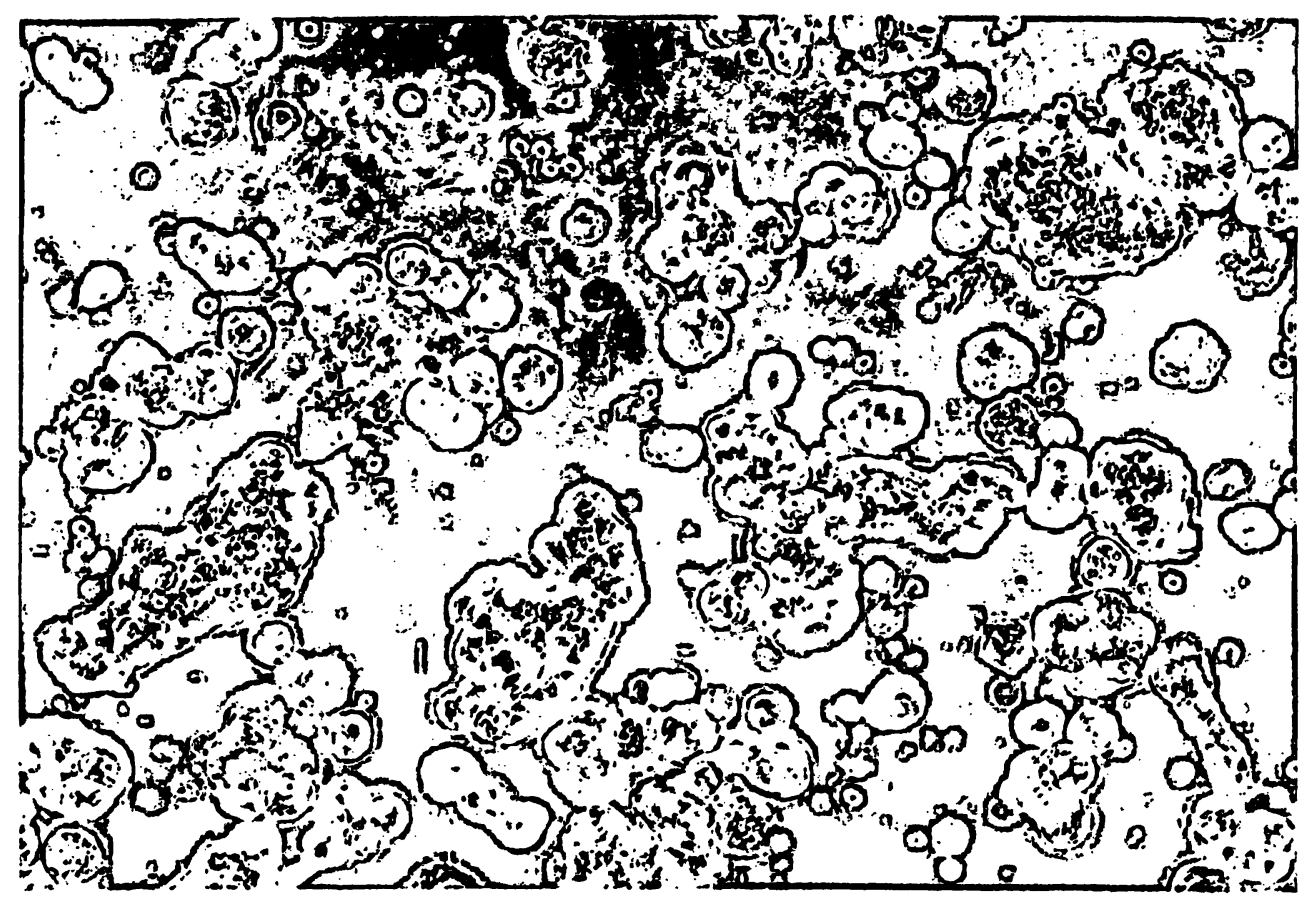

Abb. 2. Phasenkontrastmikroskopische Aufnahme isolierter Tubulusfragmente aus menschlichen Nierenrinden. Vergrößerung etwa 250 fach.

aus $10 \mathrm{mmol} / 1$ Lactat von Tubulussuspensionen bestimmt und mit denen von Schnitten verglichen. Dabei erreichten Tubulussuspensionen einen Variationskoeffizienten von $2 \%(n=126)$ und Schnitte einen Variationskoeffizienten von $29,9 \%(n=35)$.

Die Zeitabhängigkeit der Glucoseproduktion aus Pyruvat $(10 \mathrm{mmol} / \mathrm{l})$ ist in Abbildung 3 dargestellt. Bis zur 80. Minúte nahm die Glucosebildung linear zu. Nach 60 Minuten lag sie bei 3,9 $\pm 0,29 \mu \mathrm{mol} / \mathrm{g}$ Feuchtgewicht. Die Anstiege der Glucosekonzentrationen waren zwischen der 20., 40., 60. und 80. Inkubationsminute im Paarvergleich statistisch signifikant $(p<0,01$ bis 0,001).

Die Abhängigkeit der Glucoseproduktion von der Substratkonzentration zeigt Abbildung 4. Als Substrate wurde Pyruvat in Konzentrationen von 2,5; 5; 7,5 und $10 \mathrm{mmol} / \mathrm{l}$ verwendet. Aus $2,5 \mathrm{bzw} .5 \mathrm{mmol} / \mathrm{l}$ Pyruvat wurden $2,45 \pm 0,26$ bzw. $2,46 \pm 0,14 \mu \mathrm{mol} \mathrm{Glucose} / \mathrm{h} \cdot \mathrm{g}$ Feuchtgewicht gebildet; bei einer Substratkonzentration von $10 \mathrm{mmol} / 1$ waren es $3,9 \pm 0,31 \mu \mathrm{mol} / \mathrm{h} \cdot \mathrm{g}$. Die Unterschiede der Glucosesyntheseraten bei 5, 7,5 und $10 \mathrm{mmol} / 1$ Pyruvat waren im Paarvergleich statistisch signifikant $(\mathrm{p}<0,05$ und 0,02$)$.

In Abbildung 5 sind die Gluconeogeneseraten aus verschiedenen Substraten ( $10 \mathrm{mmol} / \mathrm{l})$ dargestellt. In Abwesenheit von Substrat betrug die Glucosebildung nur $0,071 \pm 0,01 \mu \mathrm{mol} / \mathrm{h} \cdot \mathrm{g}$ Feuch tgewicht. Glutamin und Prolin zeigten mit $0,5 \pm 0,25$ bzw. $1,3 \pm 0,21 \mu \mathrm{mol}$ Glucose $/ \mathrm{h} \cdot \mathrm{g}$ Feuchtgewicht die geringste Glucosebildung. Aus Pyruvat wurden 3,9 $\pm 0,21$, aus Lactat 5,2 $\pm 0,19$ $\mu \mathrm{mol}$ Glucose $/ \mathrm{h} \cdot \mathrm{g}$ Feuchtgewicht gebildet. Die höchste Syntheserate zeigte Fructose mit 11,8 $\pm 0,45 \mu \mathrm{mol}$

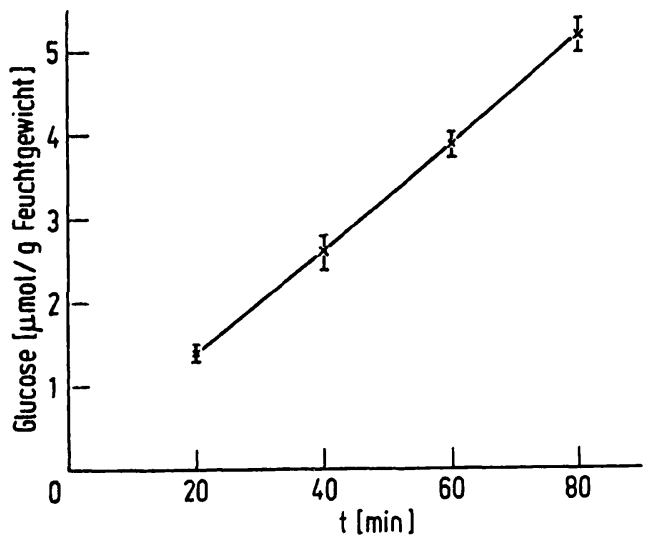

Abb. 3. Glucoseproduktion menschlicher Nierentubuli in Abhängigkcit von der Inkubationszcit.

$40 \mathrm{mg}$ Nierentubuli und Zellen wurden in $2 \mathrm{ml}$ KrebsRinger-Bicarbonat-Puffer in Gegenwart von $10 \mathrm{mmol} / 1$ Pyruvat bei $37^{\circ} \mathrm{C}$ inkubiert. Die Werte sind Mittelwerte $\left(\bar{x} \pm s_{\bar{x}}\right)$ aus 5 Präparationen.

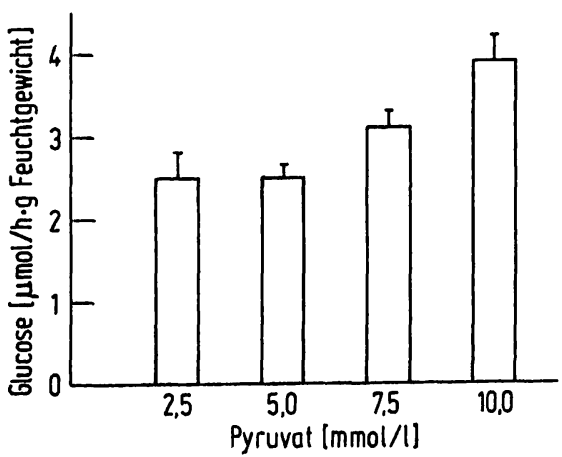

Abb. 4. Glucoseproduktion menschlicher Nierentubuli in Gegenwart verschiedener Pyruvatkonzentrationen.

$40 \mathrm{mg}$ Nierentubuli und Zcllen wurden in $2 \mathrm{ml}$ KrebsRinger-Bicarbonat-Puffer in Gegenwart der angegebenen Pyruvatkonzentrationen 60 Minuten bei $37^{\circ} \mathrm{C}$ inkubiert. Die Werte sind Mittelwerte $(\overline{\mathrm{x}} \pm 5 \overline{\mathrm{x}})$ aus 5 Präparationen. 


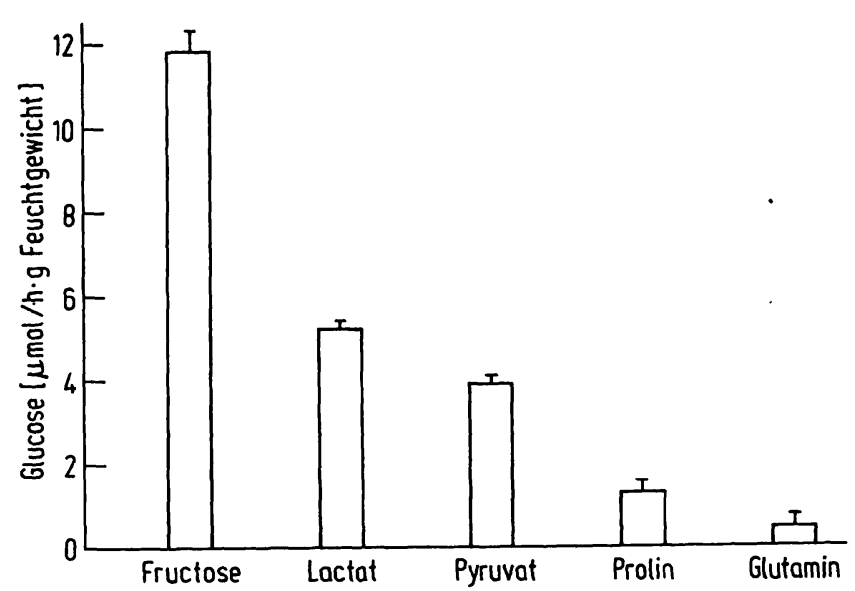

Abb. 5. Glucoseproduktion menschlicher Nierentubuli aus verschiedenen Substraten $(10 \mathrm{mmol} / \mathrm{l})$.

$40 \mathrm{mg}$ Nierentubuli und Zellen wurden in $2 \mathrm{ml}$ KrebsRinger-Bicarbonat-Puffer in Gegenwart von verschiedenen Substraten $(10 \mathrm{mmol} / \mathrm{l}) 60$ Minuten bei $37^{\circ} \mathrm{C}$ inkubiert. Die Werte sind Mittelwerte $\left(\overline{\mathrm{x}} \pm s_{\overline{\mathrm{x}}}\right)$ aus 5 Präparationen.

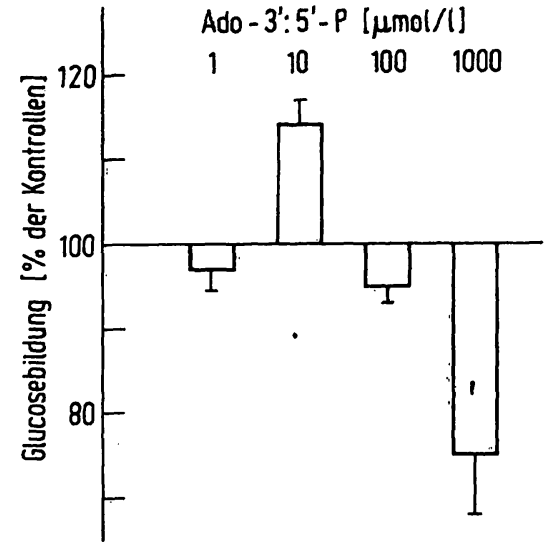

Abb. 6. Effekte verschiedener Konzentrationen von Ado- $3^{\prime}: 5^{\prime}-\mathrm{P}$ auf die Glucosebildung aus Lactat $(10 \mathrm{mmol} / \mathrm{l})$. Die Glucosebildung in den Kontrollansätzen betrug 5,17 $\pm 0,19 \mu \mathrm{mol} / \mathrm{h} \cdot \mathrm{g}$ Feuch tgewicht. $40 \mathrm{mg}$ Nierentubuli und Zellen wurden in $2 \mathrm{ml}$ Krebs-Ringer-Bicarbonat-Puffer in Gegenwart von Lactat und Ado-3':5'-P 60 Minuten bei $37^{\circ} \mathrm{C}$ inkubiert. Die Werte sind Mittelwerte $(\overline{\mathbf{x}} \pm s \overline{\mathbf{x}})$ aus 5 Präparationen.
Glucose $/ \mathrm{h} \cdot \mathrm{g}$ Feuchtgewicht. Die Unterschiede in der Glucoseproduktion zwischen Pyruvat/Lactat bzw. Lactat/Fructose waren statistisch signifikant $(\mathrm{p}<$ 0,001 bzw. 0,002):

Nierenschnitte bildeten in Gegenwart von $10 \mathrm{mmol} / 1$ Lactat mit 2,7 $\pm 0,42 \mu \mathrm{mol}$ Glucose $/ \mathrm{h} \cdot \mathrm{g}$ Feuchtgewicht signifikant $(p<0,001)$ weniger Glucose als isolierte Tubuli und Zellen.

Der Einfluß von Ado-3': $5^{\prime}$-P auf die Gluconeogenese aus Lactat $(10 \mathrm{mmol} / \mathrm{l})$ zeigt Abbildung 6 . Ohne Ado$3^{\prime}: 5^{\prime} \cdot \mathrm{P}$ wurden $5,17 \pm 0,19 \mu \mathrm{mol}$ Glucose $/ \mathrm{h} \cdot \mathrm{g}$ Feuchtgewicht gebildet. Bei einer Ado-3':5'-P Konzentration von $10 \mu \mathrm{mol} / 1$ wurde eine signifikante Steigerung $(\mathrm{p}<0,02)$ der Glucoseproduktion um $13 \%$ auf 5,99 $\pm 0,23 \mu \mathrm{mol}$ Glucose $/ \mathrm{h} \cdot \mathrm{g}$ Feuchtgewicht beobachtet. $0,1 \mathrm{mmol} / 1$ und $1 \mathrm{mmol} / 1$ Ado- $3^{\prime}: 5^{\prime}-\mathrm{P}$ hemmte die Glucosebildung um 5,2\% (p<0,02) bzw. um $25 \%$ $(\mathrm{p}<0,01)$. Bei einer Konzentration von $1 \mu \mathrm{mol} / 1$ ließ sich die Hemmung statistisch nicht sichern.

\section{Diskussion}

Durch die beschriebene Methode lassen sich Tubuli und Zellen aus Nierenrinden erwachsener Personen in hoher Ausbeute (80\%) gewinnen. Mit Trypsin und mechanischen Methoden wurden dagegen aus tierischen Nierengeweben nur Ausbeuten von etwa $28 \%$ (8) bzw. 7-10\% (9) erzielt. Darüberhinaus zerstört Trypsin Hormonrezeptoren auf der äußeren Zellmembran (10). Im Gegensatz zu der von Guder beschriebenen Isolierungsmethode für Tubuli aus Rattennieren (3) ließ sich menschliches Nierengewebe ohne eine weitgehende Zerstörung der Zellen vor Inkubation mit Kollagenase nicht durch Siebe pressen. Deshalb wurden bei unserem Verfahren die Tubuli und Zellen aus Schnitten isoliert.
Das beschriebene enzymatische Isolierungsverfahren ist in zwei Phasen gegliedert. In der ersten Phase werden die Schnitte in calciumfreiem Puffer inkubiert, da das Auswaschen von Calciumionen den Zellverband lockert. In der zweiten Phase wird Calciumchlorid zugesetzt. Calciumionen aktivieren die Kollagenase (11) und reduzieren den zellulären Kaliumverlust (12). Außerdem zeigten Zellen, die aus Rattenlebern in Anwesenheit von Calcium gewonnen wurden, höhere Sauerstoffaufnahmeraten und eine besser erhaltene mitochondriale Struktur als Zellen, die in Abwesenheit von Calcium isoliert wurden (12).

Das Muster der Snytheseraten von Glucose aus verschiedenen Substraten (Abb. 5) entspricht teilweise dem von Schnitten (13) und Tubuli (3) aus Nierenrinden von Ratten. Während allerdings bei Rattennieren die Glucosebildung aus Pyruvat höher ist als aus Lactat, findet sich bei menschlichen Tubuli ein umgekehrtes Verhältnis in den Gluconeogeneseraten. Die im Vergleich zur Rattenniere niedrigen Gluconeogeneseraten sind unseres Erachtens einerseits dadurch bedingt, daß die Patienten nur einer kurzen Nahrungskarenz (über Nacht) unterworfen waren, andererseits dürfte die Länge der Anoxiephase und die Zeit für die Anfertigung der Schnitte eine Rolle spielen. Die im Vergleich zur Pyruvatgluconeogenese höhere Glucosebildung aus Lactat wird als Hinweis dafür gewertet, daß keine größeren Lactatdehydrogenase-Verluste während der Präparation aufgetreten sind. Mechanische Isolierungsmethoden führen dagegen zu einem vollständigen Verlust mehrerer Lactatdehydrogenase-Ișoenzymfraktionen (14).

Ado-3': $5^{\prime}-\mathrm{P}$ beeinflußte in Konzentrationen von $1 \mathrm{mmol} / 1 \mathrm{bis} 10 \mu \mathrm{mol} / 1$ die Lactatgluconeogenese menschlicher Nierentubuli. Vergleichbare Effekte wurden an isolierten Nierentubuli von Ratten beobachtet (3). In Ubereinstimmung mit unseren Untersuchungen 
an menschlichen Tubuli stimulierte Ado-3': $5^{\prime}-\mathrm{P}$ in einer Konzentration von $10 \mu \mathrm{mol} / 1$ die Glucosebildung. Während die Gluconeogenese bei menschlichen Nierentubuli allerdings durch $0,1 \mathrm{mmol} / 1$ gehemmt wurde, wirkte diese Konzentration bei Tubuli von Ratten noch stimulierend. Ado- $3^{\prime}: 5^{\prime} \cdot \mathrm{P}$ in einer Konzentration von $1 \mathrm{mmol} / 1$ hemmte dagegen die Glucosebildung bei Ratte und Mensch. Die Hemmung der Lactatgluconeogenese durch höhere Konzentrationen des cyclischen Nucleotids könnte durch ein Ansteigen des Ado-5'.P Spiegels in den Tubuli hervorgerufen werden.
Dieses Reaktionsprodukt hemmt nach Weidemann, Hems \& Krebs $(15,16)$ die Gluconeogenese in der perfundierten Rattenniere und in Nierenschnitten. Die Frage, welches Hormon über Ado-3':5'-P als "second messenger" die Lactatgluconeogenese der menschlichen Niere reguliert, kann nicht sicher beantwortet werden. Untersuchungen an Rattennieren sprechen für Parathormon (17).

Diese Arbeit wurde durch Sachmittel der Deutschen Forschungsgemeinschaft gefördert.

\section{Literaturverzeichnis}

1. Burg, M. B. \& Orloff, J. (1962), Amer. J. Physiol. 203, $327-330$.

2. Berry, M. N. \& Friend, D. S. (1969), J. Cell Biol. 43, 506-520.

3. Guder, W. \& Wieland, O. (1971), in Regulation of Gluconeogenesis (Söling, H.-D. \& Willms, B., Hrsg.), 226-235, Georg Thieme Verlag, Stuttgart, New York, London.

4. Nagata, N. \& Rasmussen, H. (1970), Biochim. Biophys. Acta 215, 1-16.

5. Bojar, H., Balzer, K., Stannek, M., Reipen, W. \& Staib, W. (1974), Acta Endocrinol. (Copenhagen) Suppl. 184, 98

6. Ingebretsen, W. R. jr. \& Wagle, S. R. (1972), Biochem. Biophys. Res. Comm. 74, 403-410.

7. Stadie, W. C. \& Riggs, B. C. (1944), J. Biol. Chem. 154, 687-690.

8. Youngner, J. S. (1954), Proc. Soc. Exp. Biol. Med. 85, 202-205.

9. Thimmappayya, B., Ramachandra Reddy, R. \& Bhargava, P. M. (1970), Exp. Cell Res. 63, 333-340.

10. Johnson, M. E. M., Das, N. M., Butcher, F. R. \& Fain, J. N. (1972), J. Biol. Chem. 247, 3229-3235.

11. Seifter, S. \& Harper, E. (1970), in Methods in Enzymology (Colowick, S. P. \& Kaplan, N. O., Eds.), Vol XIX, 613635, Academic Press, New York and London.

12. Howard, R. B., Lee, J. C. \& Pesch, L. A. (1973), J. Cell Biol. 57, 642-658.

13. Krebs, H. A., Bennett, D. A. H., de Gasquet, P., Gascoyne, T. \& Yoshida, T. (1963), Biochem. J. 86, 22-27.

14. Thiele, K. G., Mattenheimer, H. (1966), diese Z. 4, 232-234.

15. Weidemann, M. J., Hems, D. A., Krebs, H. A. (1969), Biochem. J. 115, 1-10.

16. Weidemann, M. J., Hems, D. A., Krebs, H. A. (1969), Nephron 6, 282-296.

17. Pagliara, A. S., Goodmann, A. D. (1969), J. Clin. Invest. $48,1408-1412$.

Dr. H. Bojar

Institut für Physiologische Chemie II Universität Düsseldorf

4 Düsseldorf

Moorenstraße 5 
, 